\title{
OPEN Impact of poor glycemic control upon clinical outcomes after radical prostatectomy in localized prostate cancer
}

\author{
Hakmin Lee ${ }^{1,2}$, Seok-Soo Byun ${ }^{1,2}$, Sang Eun Lee ${ }^{1,2}$ \& Sung Kyu Hong ${ }^{1,2 \varpi ~}$
}

To evaluate the clinical impact of preoperative glycemic status upon oncological and functional outcomes after radical prostatectomy in patients with localized prostate cancer, we analyzed the data of 2664 subjects who underwent radical prostatectomy with preoperative measurement of hemoglobin A1c within 6 months before surgery. The possible association between high hemoglobin A1c ( $\geq 6.5 \mathrm{ng} / \mathrm{dL}$ ) and oncological/functional outcomes was evaluated. Among all subjects, 449 (16.9\%) were categorized as the high hemoglobin A1c group and 2215 (83.1\%) as the low hemoglobin A1c group. High hemoglobin A1c was associated with worse pathological outcomes including extra-capsular extension (HR 1.277,95\% Cl 1.000-1.630, $p=0.050$ ) and positive surgical margin ( $\mathrm{HR} 1.302,95 \% \mathrm{Cl} 1.012-1.674, p=0.040$ ) in multi-variate regression tests. Kaplan-Meier analysis showed statistically shorter biochemical recurrence-free survival in the high hemoglobin A1c group $(p<0.001)$, and subsequent multivariate Cox proportional analyses revealed that high hemoglobin A1c is an independent predictor for shorter BCR-free survival ( $\mathrm{HR} 1.135,95 \% \mathrm{Cl} 1.016-1.267, p=0.024)$. Moreover, the high hemoglobin A1c group showed a significantly longer incontinence-free survival than the low hemoglobin A1c group $(p=0.001)$, and high preoperative hemoglobin A1c was also an independent predictor for longer incontinence-free survival in multivariate Cox analyses (HR 0.929, $95 \% \mathrm{Cl} 0.879-0.981, p=0.008$ ). The high preoperative hemoglobin A1c level was independently associated with worse oncological outcomes and also with inferior recovery of urinary continence after radical prostatectomy.

Diabetes mellitus (DM) is one of the most prevalent and disabling chronic diseases in many developed countries, including the United States ${ }^{1}$. DM is a well-known risk factor for several serious diseases such as stroke, heart attack, vision impairment, peripheral vascular disease, and even depression ${ }^{2}$. Moreover, DM is also associated with cancer development and aggressive clinical behaviors ${ }^{3}$. However, the relationship between DM and prostate cancer $(\mathrm{PCa})$ seems to be more complicated ${ }^{4}$. Unlike most other cancers, which usually have positive relationship between DM and the development of cancer, several previous meta-analyses have shown an inverse relationship between $\mathrm{DM}$ and $\mathrm{PCa}^{5-6}$. Previously, we investigated the impact of DM on oncological outcomes in patients treated with radical prostatectomy (RP) for localized $\mathrm{PCa}^{7}$. However, we could not find any significant associations between DM and clinico-pathologic outcomes after RP. Conversely, we observed that poor glycemic control, which was represented by hemoglobin A1c (HbA1c) level, was significantly associated with some adverse pathologic features, including high Gleason score and extra-capsular extension among the patients with DM. Subsequently, another study reported similar findings after analyzing the Shared Equal Access Regional Cancer Hospital database after surgery ${ }^{8}$. In their study, HbAlc tertile was predictive of a higher pathologic Gleason score $(p=0.001)$. However, their study was limited due to the small sample size $(\mathrm{n}=247)$. Despite the interesting results of these two studies, they only analyzed the impact of preoperative glycemic control in patients who were already diagnosed with DM but not in those who did not have prior DM diagnosis. As several other studies also showed that the preoperative glycemic control was associated with worse prognosis in other malignancies, including colorectal and liver cancers, the impact of glycemic control can also be prognostic in patients with PCa regardless

${ }^{1}$ Department of Urology, Seoul National University Bundang Hospital, 82 Gumi-ro, 173 Beon-gil, Bundang-gu, Seongnam-si 463-707, Gyeonggi-do, Korea. ${ }^{2}$ Department of Urology, Seoul National University College of Medicine, Seoul, Korea. ${ }^{\circledR}$ email: skhong@snubh.org 
of previous DM history ${ }^{9-10}$. Therefore, we tried to evaluate the clinical impact of preoperative glycemic control on the oncological and functional outcomes in patients who were treated with RP for localized PCa.

\section{Materials and methods}

After obtaining approval from Seoul National University Bundang Hospital Institutional Review Board, we retrospectively analyzed the data of 2716 subjects who were diagnosed with localized PCa and subsequently treated with RP between January 2010 and December 2018. All analyses were performed in accordance with the guidelines and regulations of our institutional review board and informed consent was waived due to the nature of retrospective analyses and minimal risk to the participants. After the additional exclusion of 52 patients (preoperative androgen deprivation therapy $[n=18]$, previous pelvic radiation $[n=3]$, and incomplete information $[n=31]$ ), we finally analyzed the data of 2664 patients. Clinical and pathologic information was retrieved from our prospectively maintained institutional database. HbAlc measurements were routinely performed as part of the preoperative work-up. The history of DM was verified by self-administered questionnaires at the time of admission for surgery, which asked if they had been diagnosed with DM during their lifetime. Patients who were diagnosed with DM before surgery were considered to have a history of DM. The type of surgery (open/robotic/ laparoscopic), degree of neurovascular bundle preservation and lymph node dissection were decided by each surgeon's clinical opinion and/or counseling with patients. The measurement of $\mathrm{HbAlc}$ was routinely performed as a part of preoperative work-up for general anesthesia in this study. To determine the optimal cutoff value for $\mathrm{HbAlc}$, the receiver operating curve of $\mathrm{HbAlc}$ on BCR was analyzed. Since the HbAlc value of $6.5 \mathrm{ng} / \mathrm{dL}$ showed the largest Youden's index, the cutoff value was set at $6.5 \mathrm{ng} / \mathrm{dL}$. Therefore, patients with HbA1c $<6.5 \mathrm{ng} / \mathrm{dL}$ were categorized as the low HbA1c group, while the others were categorized as the high HbA1c group. Pathologic outcomes such as seminal vesicle invasion, an extraprostatic extension of tumor, positive surgical margin, and lymph node invasion were inspected as previously described ${ }^{7}$. BCR was defined as a prostate-specific antigen (PSA) level $\geq 0.2 \mathrm{ng} / \mathrm{mL}$ in two consecutive tests as per the guidelines of the American Urological Association ${ }^{11}$. The follow-up period was defined as months from the date of surgery to the date of the last visit or mortality. After surgery, patients were usually followed up at an interval of 3- to 6-months during the initial two years and yearly thereafter, when there was no evidence of BCR. The functional outcomes after surgery were evaluated using a patient-reported questionnaire, including the Expanded Prostate Cancer Index Composite for Clinical Practice and International Index of Erectile Function (IIEF)-5. The recovery of urinary continence was defined when the amount of pad usage was counted under one pad/day, and erectile dysfunction was defined when the IIEF-5 score was under 22.

To compare the clinical characteristics between the subgroups, chi-square, and Student's t-tests were performed. Multivariate binomial regression tests were performed to evaluate possible associations of high HbA1c levels with adverse pathological outcomes. To analyze survival outcomes, Kaplan-Meier analysis and Cox proportional hazard model were utilized. All statistical analyses were performed using SPSS software (SPSS 22.0, Chicago, IL, USA). All $p$ values are presented as two-sided values, and $p<0.05$ was considered statistically significant.

\section{Results}

The clinical and pathological characteristics of all patients are summarized in Table 1 . The median age was 67.0 (interquartile range [IQR] 62.0-72.0) years and median PSA was 7.4 (5.0-12.4) ng/dL. Among entire patients, $1184(44.4 \%)$ patients had radical prostatectomy with neurovascular bundle preservation. There were $124(4.6 \%)$ patients who had positive lymph node invasion from surgical pathology and $164(6.2 \%)$ patients had salvage therapy after sugery. There were $2215(83.1 \%)$ patients in the low HbA1c group and $449(16.9 \%)$ in the high HbAlc group. When we compared the distribution of clinicopathological characteristics between the two subgroups, the prevalence of DM and hypertension was significantly higher in the high HbAlc group than in the low HbA1c group (all $p<0.001$ ). The high HbAlc group also showed significantly unfavorable clinical features in terms of preoperative PSA, biopsy grade group, clinical stage, pathologic grade group, and pathologic stage (all $p<0.05)$. Similarly, adverse pathologic outcomes such as positive surgical margin $(p=0.003)$, seminal vesicle invasion $(p=0.032)$, and extra-capsular extension $(p=0.001)$ were also more prevalent in the high HbAlc group. Multivariate regression analyses showed that $\mathrm{HbA1c}$ was independently associated with extracapsular extension (HR 1.277, 95\% CI 1.000-1.630, $p=0.050$ ) and positive surgical margin (HR 1.302, 95\% CI 1.02-1.674, $p=0.040$ ), respectively (Table 2).

The total medial follow-up time of all subjects was 23 months (IQR, 6.0-47.0). After a median follow-up time of 8 (IQR 6.0-23.0) months, 443 (16.6\%) patients developed BCR [329 (14.9\%) subjects in low HbA1c group, 114 (25.4\%) in high HbA1c group]. Among them, there were 172 (6.5\%) patients who had persistent-detectable PSA. The Kaplan-Meier analyses showed that the high HbA1c group had a significantly shorter BCR-free survival than the low HbA1c group $(p<0.001)$ (Fig. 1). The subsequent multivariate Cox proportional hazard analyses revealed that a high $\mathrm{HbAlc}$ level was an independent predictor for shorter BCR-free survival (HR 1.135, 95\% CI $1.016-1.267, p=0.024)$, while the preoperative history for DM did not show any statistically significant results (Table 3, Supplementary Table 1).

Among all subjects, 2041 (76.6\%) patients completed questionnaires about functional outcomes. Among them, 99 (4.9\%) patients still had incontinence after a median follow-up of 31 (IQR, 12.0-51.0) months. The Kaplan-Meier analyses showed that the high HbAlc group showed significantly longer incontinence-free survival $(p=0.008)$ (Fig. 2). Subsequent Cox proportional hazard analysis revealed that a high HbA1c level was an independent predictor for longer incontinence-free survival (HR 0.929, 95\% CI 0.879-0.981, $p=0.008$ ) when analyzed as a continuous variable. A similar result was observed when HbA1c was adjusted as a categorical variable (HR 0.862, 95\% CI 0.765-0.673, $p=0.016$ ) (Table 4). Among all patients, $312(11.7 \%)$ patients had no erectile dysfunction before surgery. When we compared the erectile dysfunction-free survival between the high and 


\begin{tabular}{|c|c|c|c|c|}
\hline Median (IQR) or number (percent) & $\begin{array}{l}\text { Entire patients } \\
(\mathrm{n}=2664)\end{array}$ & $\begin{array}{l}\text { High HbAlc group } \\
(\mathrm{n}=449)\end{array}$ & $\begin{array}{l}\text { Low HbAlc group } \\
(\mathbf{n}=2215)\end{array}$ & $p$ value \\
\hline Age (y) & $67.0(62.0-72.0)$ & $68.0(63.0-72.0)$ & $67.0(61.0-71.0)$ & 0.171 \\
\hline BMI $\left(\mathrm{kg} / \mathrm{m}^{2}\right)$ & $24.6(22.9-26.3)$ & $25.1(23.3-26.7)$ & $24.5(22.9-26.2)$ & 0.897 \\
\hline Diabetes mellitus & $22.3 \%$ & $75.5 \%$ & $11.5 \%$ & $<0.001$ \\
\hline Hypertension & $49.0 \%$ & $59.2 \%$ & $46.9 \%$ & $<0.001$ \\
\hline PSA & $7.4(5.0-12.4)$ & $7.9(5.3-14.0)$ & $7.3(5.0-12.0)$ & $<0.001$ \\
\hline Prostate volume & $33.5(26.0-42.5)$ & $34.4(27.0-44.0)$ & $33.0(26.0-42.0)$ & 0.007 \\
\hline Biopsy grade group & & & & 0.035 \\
\hline Group 1 & $28.9 \%$ & $24.3 \%$ & $29.8 \%$ & \\
\hline Group 2 & $31.4 \%$ & $29.9 \%$ & $31.7 \%$ & \\
\hline Group 3 & $18.8 \%$ & $21.9 \%$ & $18.1 \%$ & \\
\hline Group 4 & $16.2 \%$ & $17.6 \%$ & $15.9 \%$ & \\
\hline Group 5 & $4.7 \%$ & $6.3 \%$ & $4.4 \%$ & \\
\hline Clinical stages & & & & 0.202 \\
\hline cT1 & $53.0 \%$ & $51.0 \%$ & $53.4 \%$ & \\
\hline cT2 & $27.1 \%$ & $30.5 \%$ & $26.5 \%$ & \\
\hline cT3 & $19.9 \%$ & $18.5 \%$ & $20.2 \%$ & \\
\hline Pathologic grade group & & & & 0.028 \\
\hline Group 1 & $4.6 \%$ & $5.1 \%$ & $4.5 \%$ & \\
\hline Group 2 & $42.4 \%$ & $36.6 \%$ & $43.6 \%$ & \\
\hline Group 3 & $35.7 \%$ & $36.6 \%$ & $35.5 \%$ & \\
\hline Group 4 & $6.5 \%$ & $7.8 \%$ & $6.2 \%$ & \\
\hline Group 5 & $10.8 \%$ & $13.8 \%$ & $10.2 \%$ & \\
\hline Pathologic stages & & & & 0.041 \\
\hline pT2 & $9.5 \%$ & $7.4 \%$ & $9.9 \%$ & \\
\hline$\geq \mathrm{pT3}$ & $90.5 \%$ & $92.7 \%$ & $90.1 \%$ & \\
\hline ECE & $31.6 \%$ & $38.4 \%$ & $30.2 \%$ & 0.001 \\
\hline SVI & $12.1 \%$ & $15.2 \%$ & $11.5 \%$ & 0.032 \\
\hline PSM & $21.2 \%$ & $26.6 \%$ & $20.1 \%$ & 0.003 \\
\hline
\end{tabular}

Table 1. Summarization of clinico-pathologic characteristics according to the hemoglobin A1c level. $I Q R$, interquartile range, $B M I$ body mass index, PSA prostate specific antigen, ECE extracapsular extension, $S V I$ seminal vesicle invasion, $P S M$ positive surgical margin.

\begin{tabular}{|l|l|l|l|}
\hline & HR & $\mathbf{9 5 \%}$ CI of HR & $\boldsymbol{p}$ value \\
\hline Pathologic Gleason score $(\geq 4+4)$ & 1.279 & $0.976-1.675$ & 0.075 \\
\hline Pathologic Stage $(\geq \mathrm{T} 3)$ & 1.251 & $0.847-1.849$ & 0.260 \\
\hline Extracapsular extension & 1.277 & $1.000-1.630$ & 0.050 \\
\hline Seminal vesicle invasion & 1.114 & $0.789-1.573$ & 0.541 \\
\hline Positive surgical margin & 1.302 & $1.012-1.674$ & 0.040 \\
\hline
\end{tabular}

Table 2. Multivariate analyses for the impact of high $\mathrm{HbA1c}$ level on aggressive pathologic outcomes. $H b A 1 c$ hemoglobin A1c, HR hazard ratio, CI confidence interval. Multivariate analyses were adjusted for age, prostatic specific antigen, body mass index, prostate volume, pathologic Gleason score and pathologic stage.

low HbAlc groups in those patients who were potent before RP, the high HbAlc group showed longer erectile dysfunction-free survival, but this result was not statistically significant ( $p=0.080)$ (Fig. 3).

\section{Discussion}

In the present study, we observed that the preoperative HbAlc level was significantly associated with worse clinical characteristics and adverse pathological outcomes in patients who were treated with RP for PCa. Furthermore, a high $\mathrm{HbA1c}$ level was found to be a statistically significant predictor for shorter postoperative BCR-free survival, whereas a previous history of DM did not show any significant associations. In addition to the oncological outcomes, the preoperative $\mathrm{HbA1c}$ level was associated with functional outcomes after surgery, specifically in the recovery of urinary continence. The recovery of erectile function was also better in the low HbAlc group, but the results were not statistically significant in this study. 


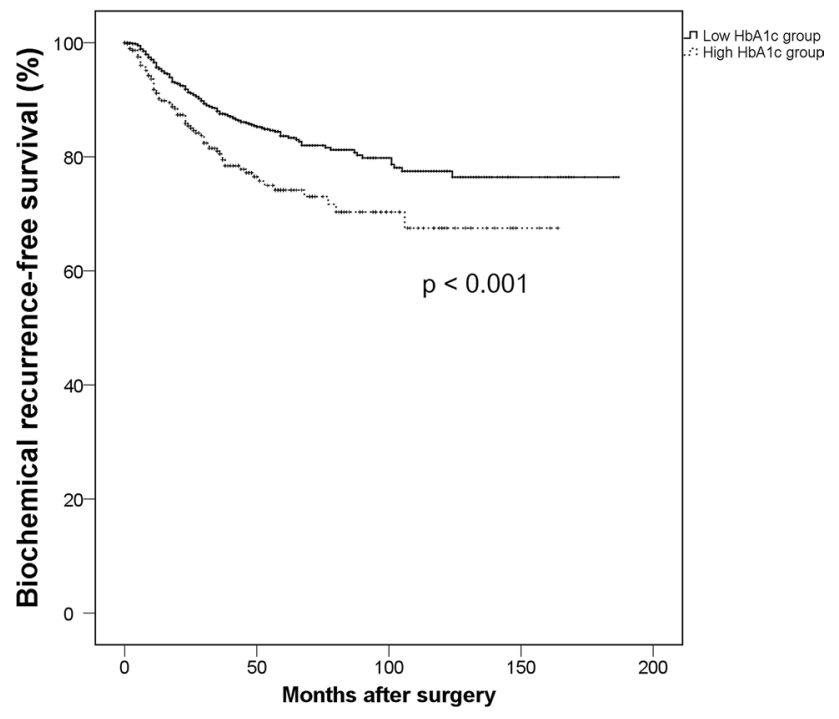

Figure 1. Kaplan-Meier analyses on biochemical recurrence-free survival according to the preoperative HbAlc.

\begin{tabular}{|c|c|c|c|c|c|c|}
\hline & \multicolumn{3}{|c|}{ When adjusted by history of DM } & \multicolumn{3}{|c|}{ When adjusted by HbA1c level } \\
\hline & HR & 95\% CI & p value & HR & 95\% CI & $p$ value \\
\hline Age & 0.978 & $0.962-0.995$ & 0.013 & 0.978 & $0.962-0.995$ & 0.012 \\
\hline BMI & 1.006 & $0.972-1.043$ & 0.719 & 1.004 & $0.966-1.043$ & 0.845 \\
\hline History of DM & 1.043 & $0.794-1.371$ & 0.760 & & Not included & \\
\hline HbAlc & & Not included & & 1.135 & $1.016-1.267$ & 0.024 \\
\hline PSA & 1.003 & $1.001-1.005$ & 0.017 & 1.003 & $1.000-1.005$ & 0.024 \\
\hline Prostate volume & 0.998 & $0.988-1.008$ & 0.743 & 0.998 & $0.988-1.008$ & 0.715 \\
\hline \multicolumn{7}{|l|}{ Pathologic grade group } \\
\hline Group 1 & & Reference & & & Reference & \\
\hline Group 2 & 3.715 & $0.895-15.416$ & 0.071 & 3.754 & $0.905-15.576$ & 0.068 \\
\hline Group 3 & 18.148 & $4.429-74.357$ & $<0.001$ & 18.012 & $4.397-73.788$ & $<0.001$ \\
\hline Group $4-5$ & 39.749 & $9.606-164.487$ & $<0.001$ & 40.121 & $9.700-165.954$ & $<0.001$ \\
\hline Pathologic stage ( $\geq \mathrm{pT} 3)$ & 2.678 & $1.256-5.707$ & 0.011 & 2.674 & $1.255-5.698$ & 0.011 \\
\hline PSM & 2.811 & $2.185-3.618$ & $<0.001$ & 2.797 & $2.172-3.600$ & $<0.001$ \\
\hline Lymph node invasion & 1.674 & $0.855-3.277$ & 0.133 & 1.747 & $0.894-3.412$ & 0.103 \\
\hline
\end{tabular}

Table 3. Multivariate analyses using Cox proportional hazard model on biochemical recurrence. BMI Body mass index, DM diabetes mellitus, HbA1c hemoglobin A1c, PSA prostate specific antigen, PSM positive surgical margin.

Previously, DM has been reported to be associated with an increased risk of various cancers, including colo-rectum, breast, pancreas, endometrial, liver, and bladder ${ }^{12-17}$. However, the relationship between DM and PCa has been regarded as an inverse relationship ${ }^{18-19}$. The unique inverse association between DM and PCa development has not been fully understood, but previous studies suggested some possible explanations, including the detection bias from regular checkups, hormonal differences, and metabolic alterations associated with DM. Moreover, some studies demonstrated that DM was related to worse survival outcomes in PCa despite the inverse relationship between DM and PCa development ${ }^{20-22}$. Lee et al. performed meta-analysis with the data of 274,677 subjects from 17 cohort studies and found that there was a $29 \%$ increase in cancer-specific death in patients with DM (RR 1.29, 9\% CI 1.22-1.38, I2=66.68\%) than in others without $\mathrm{DM}^{21}$. On the other hand, other studies demonstrated no significant associations between DM and survival of $\mathrm{PCa}^{23-25}$. In the present study, we observed that the simple previous history of DM was not statistically related to postoperative recurrence after a surgical treatment for localized PCa. However, the status of preoperative glycemic control, which was represented by the $\mathrm{HbA1c}$ level, showed significant associations with postoperative oncological outcomes.

Not many studies have reported on the impact of glycemic control on the outcome of PCa independently with a history of DM. Gapstur et al. previously reported that hyperglycemia was significantly associated with increased mortality for PCa after analyzing 20,433 subjects who underwent health screening examination ${ }^{26}$. In 


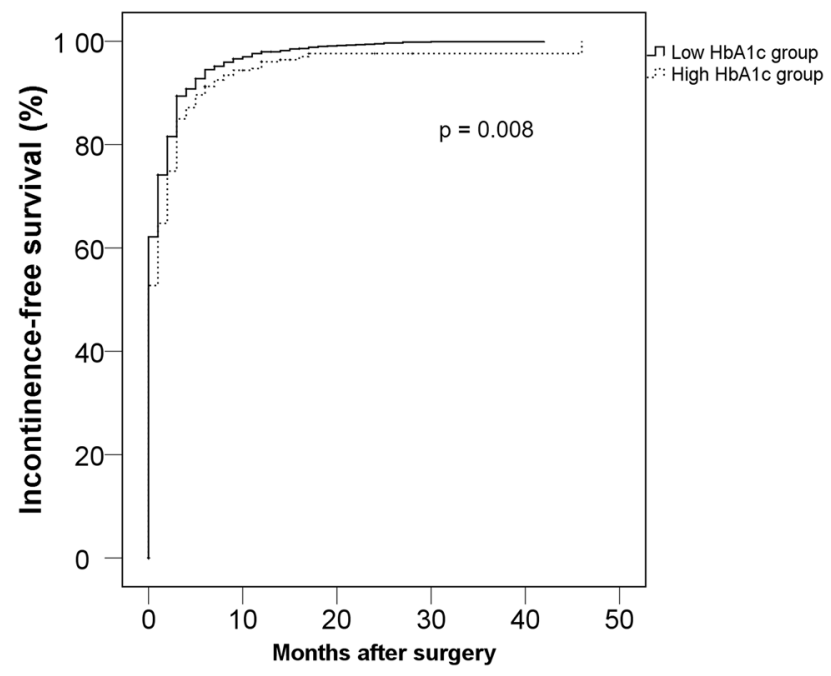

Figure 2. Kaplan-Meier analyses on the recovery of urinary incontinence according to the preoperative HbAlc.

\begin{tabular}{|l|l|l|l|l|l|l|l|}
\hline \multirow{2}{*}{} & \multicolumn{3}{|l|}{ HbA1c as continuous variable } & \multicolumn{3}{l|}{ HbA1c as categorical variable } \\
\cline { 2 - 8 } & HR & $\mathbf{9 5 \%}$ CI & p value & HR & $\mathbf{9 5 \%}$ CI & p value \\
\hline Age & 0.992 & $0.985-0.998$ & 0.016 & 0.992 & $0.985-0.998$ & 0.016 \\
\hline PSA & 1.002 & $0.984-1.020$ & 0.853 & 1.002 & $0.984-1.020$ & 0.827 \\
\hline Prostate volume & 1.000 & $0.998-1.002$ & 0.972 & 1.000 & $0.998-1.002$ & 0.946 \\
\hline HbA1c & 1.000 & $0.997-1.003$ & 0.935 & 1.000 & $0.997-1.004$ & 0.903 \\
\hline High pathologic grade group $(\geq$ group 4$)$ & 1.098 & $0.963-1.252$ & 0.162 & 1.103 & $0.968-1.258$ & 0.142 \\
\hline High pathologic stage $(\geq 3)$ & 1.023 & $0.879-1.192$ & 0.766 & 1.024 & $0.879-1.192$ & 0.765 \\
\hline Neurovascular bundle preservation & & & & & & \\
\hline None & & Reference & & & Reference & \\
\hline Unilateral / partial & 1.121 & $0.929-1.354$ & 0.234 & 1.098 & $0.904-1.335$ & 0.346 \\
\hline Bilateral & 1.184 & $1.058-1.326$ & 0.003 & 1.147 & $1.020-1.290$ & 0.022 \\
\hline
\end{tabular}

Table 4. Multivariate analyses using Cox proportional hazard model on continence-free survival. BMI Body mass index, DM diabetes mellitus, HbA1c hemoglobin A1c, PSA prostate specific antigen, PSM positive surgical margin.

addition, another study by Ma et al. found that men with high C-peptide (an insulin surrogate) had a $>2$-fold increased risk of PCa-specific mortality than those with low C-peptide ${ }^{27}$. More recently, Farnoosh et al. analyzed 1,502 subjects who had DM history with HbA1c measurements before RP after analyzing the Shared Equal Access Regional Cancer Hospital database ${ }^{28}$. They found that high HbAlc was significantly associated with metastasis (HR 1.21, 95\% CI 1.02. 1.44, $p=0.031$ ) and progression to castration-resistant PCa (HR 1.27, 95\% CI 1.03-1.56, $p=0.023$ ). However, these studies investigated the relationship between $\mathrm{HbA1c}$ level and PCa outcomes only in patients with a history of DM but not in those without DM history. In the present study, we measured HbAlc as a routine preoperative work-up regardless of a previous history of DM and tried to evaluate the clinical influence of glycemic status on the postoperative outcomes. We observed that preoperative glycemic control was associated with postoperative oncological outcomes, but not with prior history of DM diagnosis.

It should be noted that the preoperative $\mathrm{HbAlc}$ level was also related to better urinary function recovery after surgery, in our study. We are not the first to evaluate the influence of DM and/or glycemic status on functional recovery after RP. Teber et al. previously reported that the history of type $2 \mathrm{DM}$ was a strong predictor of postoperative incontinence in their retrospective analyses of 2071 patients after laparoscopic $\mathrm{RP}^{29}$. However, their study was limited by the small number of subjects, as there were only 135 patients with type 2 DM in the study. Considering that the current DM prevalence is approximately $10.5 \%$ for the overall population and even higher in the elderly, their study might have been biased due to some selection bias and/or recall bias. Furthermore, they performed a logistic regression test without considering the time interval between surgery and recovery of incontinence. In the present study, we compared the impact of glycemic control on postoperative incontinence with consideration of time onset for exact comparison. High preoperative HbAlc was revealed to be an independent predictor for worse recovery of postoperative incontinence, both as categorical and continuous variables. 


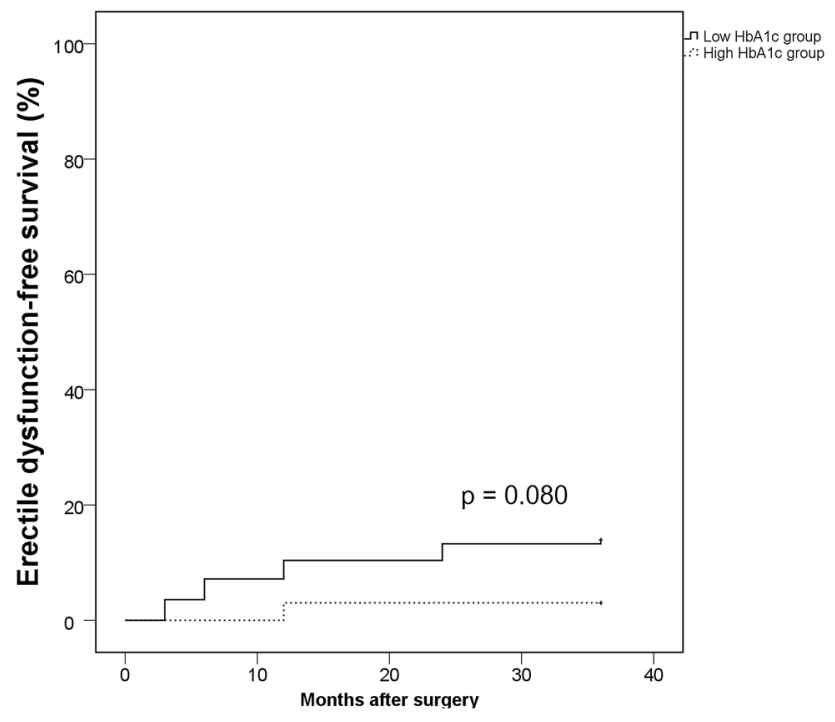

Figure 3. Kaplan-Meier analyses on the recovery of erectile dysfunction according to the preoperative HbA1c.

On the other hand, the recovery for erectile dysfunction was also superior in the low HbAlc group than in the high HbAlc group in our study, but the result was not statistically significant $(p=0.080)$. We believe that the influence of $\mathrm{HbAlc}$ on erectile dysfunction should be reevaluated in future studies because our study could not analyze a sufficient number of subjects who had normal erectile function before surgery.

We acknowledge that there may be limitations to our study, including the retrospective study design. Moreover, the main limitation of this study is that we could only analyze the level of HbA1c and not of the other hormones related to glycemic control, such as insulin or glucagon. Another limitation is that the glycemic control was only estimated by a single preoperative measurement, not by several postoperative follow-ups. As the glycemic status can vary according to the different time points and patients' postoperative clinical status, the single measurement cannot be the exact representative for patients' glycemic status. And more importantly, there is a possibility for selection bias in the present study as we only included the patients who were treated by radical prostatectomy. Therefore, patients who cannot tolerate the surgery for poor general condition or patients with locally-advanced disease were not included our study. Another limitation for our study is that we could not analyze the influence of salvage treatment after BCR as the salvage treatment can also affect the postoperative functional outcomes. Finally, we could not analyze the influence of usage for phosphodiesterase type 5 inhibitor and other general conditions which can affect the level of HbAlc. Therefore, our findings should be re-tested in future studies with prospective design and longer follow-up.

\section{Conclusions}

A worse preoperative glycemic status, represented by the HbA1c level, was clinically associated with inferior oncological outcomes after surgical treatment of localized PCa. The recovery of urinary incontinence was also significantly inferior in the subgroup with worse preoperative glycemic status. On the other hand, the history of DM did not show any clinical association with oncological and functional outcomes after RP.

Received: 19 January 2021; Accepted: 20 May 2021

Published online: 07 June 2021

\section{References}

1. Centers for Disease Control and Prevention. Atlanta, GA: U.S. Department of Health and Human Services, Centers for Disease Control and Prevention; National diabetes fact sheet: General information and national estimates on diabetes in the United States. www.cdc.gov/diabetes/pubs/estimates.htm3 (2003).

2. Nathan, D. M. Long-term complications of diabetes mellitus. N. Engl. J. Med. 328, 1676-1683 (1993).

3. Abudawood, M. Diabetes and cancer: A comprehensive review. J. Res. Med. Sci. 25(24), 94 (2019).

4. Kasper, J. S., Liu, Y. \& Giovannucci, E. Diabetes mellitus and risk of prostate cancer in the health professionals follow-up study. Int. J. Cancer 124(6), 1398-1403 (2009).

5. Jian Gang, P., Mo, L., Lu, Y., Runqi, L. \& Xing, Z. Diabetes mellitus and the risk of prostate cancer: An update and cumulative meta-analysis. Endocr. Res. 40(1), 54-61 (2015).

6. Bonovas, S., Filioussi, K. \& Tsantes, A. Diabetes mellitus and risk of prostate cancer: A meta-analysis. Diabetologia 47(6), 1071-1078 (2004).

7. Hong, S. K. et al. Significance of preoperative HbA1c level in patients with diabetes mellitus and clinically localized prostate cancer. Prostate 69(8), 820-826 (2009).

8. Kim, H. S. et al. Glycemic control and prostate cancer progression: Results from the SEARCH database. Prostate 70(14), 1540-1546 (2010).

9. Siddiqui, A. A. et al. Elevated HbAlc is an independent predictor of aggressive clinical behavior in patients with colorectal cancer: A case-control study. Dig. Dis. Sci. 53, 2486-2494 (2008). 
10. Kaneda, K. et al. The influence of postoperative glycemic control on recurrence after curative resection in diabetics with hepatitis C virus-related hepatocellular carcinoma. J. Surg. Oncol. 105, 606-611 (2012).

11. Cookson, M. S. et al. Variation in the definition of biochemical recurrence in patients treated for localized prostate cancer: The American Urological Association Prostate Guidelines for Localized Prostate Cancer Update Panel report and recommendations for a standard in the reporting of surgical outcomes. J. Urol. 177, 540-545 (2007).

12. Larsson, S. C., Orsini, N. \& Wolk, A. Diabetes mellitus and risk of colorectal cancer: A meta-analysis. J. Natl. Cancer Inst. 97(22), 1679-1687 (2005).

13. Larsson, S. C., Mantzoros, C. S. \& Wolk, A. Diabetes mellitus and risk of breast cancer: A meta-analysis. Int. J. Cancer 121(4), 856-862 (2007).

14. Everhart, J. \& Wright, D. Diabetes mellitus as a risk factor for pancreatic cancer: A meta-analysis. Int. J. Cancer 121(4), 856-862 (2007).

15. Friberg, E., Orsini, N., Mantzoros, C. S. \& Wolk, A. Diabetes mellitus and risk of endometrial cancer: a meta-analysis. Diabetologia 50(7), 1365-1374 (2006)

16. Adami, H. O. et al. Excess risk of primary liver cancer in patients with diabetes mellitus. J. Natl. Cancer Inst. 88(20), 1472-1477 (1996).

17. Larsson, S. C., Orsini, N., Brismar, K. \& Wolk, A. Diabetes mellitus and risk of bladder cancer: A meta-analysis. Diabetologia 49(12), 2819-2823 (2006).

18. Adami, H. O. et al. Cancer risk in patients with diabetes mellitus. Cancer Causes Control 2, 307-314 (1991).

19. Giovannucci, E., Rimm, E. B., Stampfer, M. J., Colditz, G. A. \& Willett, W. C. Diabetes mellitus and risk of prostate cancer. Cancer Causes Control 9(1), 3-9 (1998).

20. Will, J. C., Vinicor, F. \& Calle, E. E. Is diabetes mellitus associated with prostate cancer incidence and survival?. Epidemiology 10, 313-318 (1999).

21. Lee, J., Giovannucci, E. \& Jeon, J. Y. Diabetes and mortality in patients with prostate cancer: A meta-analysis. Springerplus 5(1), 1548 (2016).

22. Hirata, Y. et al. Prognostic significance of diabetes mellitus and dyslipidemia in men receiving androgen-deprivation therapy for metastatic prostate cancer. Prostate Int. 7(4), 166-170 (2019).

23. Rieken, M. et al. Association of diabetes mellitus and metformin use with biochemical recurrence in patients treated with radical prostatectomy for prostate cancer. World J. Urol. 32, 999-1005 (2014).

24. Jayachandran, J. et al. Diabetes and outcomes after radical prostatectomy: are results affected by obesity and race? Results from the shared equal-access regional cancer hospital database. Cancer Epidemiol. Biomarkers Prev. 19, 9-17 (2010).

25. Wu, C. et al. Diabetes predicts metastasis after radical prostatectomy in obese men: Results from the SEARCH database. BJU Int. 111(8), E310-E318 (2013).

26. Gapstur, S. M. et al. Postload plasma glucose concentration and 27-year prostate cancer mortality (United States). Cancer Causes Control 12, 763-772 (2001).

27. Ma, J. et al. Prediagnostic body-mass index, plasma C-peptide concentration, and prostate cancer-specific mortality in men with prostate cancer: A longterm survival analysis. Lancet Oncol. 9, 1039-1047 (2008).

28. Nik-Ahd, F. et al. Poorly controlled diabetes increases the risk of metastases and castration-resistant prostate cancer in men undergoing radical prostatectomy: Results from the SEARCH database. Cancer 125(16), 2861-2867 (2019).

29. Teber, D. et al. Is type 2 diabetes mellitus a predictive factor for incontinence after laparoscopic radical prostatectomy? A matched pair and multivariate analysis. J. Urol. 183(3), 1087-1091 (2010).

\section{Author contributions}

H.L.: Data analysis, Data collection and management, manuscript writing/editing. S.-S.B.: project development, manuscript editing. S.E.L.: project development, manuscript editing. S.K.H.: project development, manuscript writing/editing. All authors reviewed the manuscript.

\section{Competing interests}

The authors declare no competing interests.

\section{Additional information}

Supplementary Information The online version contains supplementary material available at https://doi.org/ 10.1038/s41598-021-91310-3.

Correspondence and requests for materials should be addressed to S.K.H.

Reprints and permissions information is available at www.nature.com/reprints.

Publisher's note Springer Nature remains neutral with regard to jurisdictional claims in published maps and institutional affiliations.

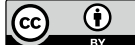

Open Access This article is licensed under a Creative Commons Attribution 4.0 International License, which permits use, sharing, adaptation, distribution and reproduction in any medium or format, as long as you give appropriate credit to the original author(s) and the source, provide a link to the Creative Commons licence, and indicate if changes were made. The images or other third party material in this article are included in the article's Creative Commons licence, unless indicated otherwise in a credit line to the material. If material is not included in the article's Creative Commons licence and your intended use is not permitted by statutory regulation or exceeds the permitted use, you will need to obtain permission directly from the copyright holder. To view a copy of this licence, visit http://creativecommons.org/licenses/by/4.0/.

(C) The Author(s) 2021 